\title{
Design and Optimization Strategies for Muscle-like Direct Drive Linear Permanent Magnet Motors
}

\author{
Bryan P. Ruddy and Ian W. Hunter \\ BioInstrumentation Laboratory \\ Depatment of Mechanical Engineering \\ Massachusetts Institute of Technology \\ Cambridge, Massachusetts 02139-4301 \\ Email: \{ruddy, ihunter\}@mit.edu
}

\begin{abstract}
We report a new approach to the design of directdrive linear permanent magnet motors for use in general-purpose robotic actuation, with particular attention to applications in bird-scale flapping-wing robots. We show a simple, quantitative analytical modeling framework for this class of actuators, and demonstrate inherent scaling properties that allow the production of motors with force densities and efficiencies comparable to those of biological muscles. We will show how this model leads to a set of practical design specifications for muscle-like motors, and examine the resulting trade-off between thermal management and motor fabrication complexity.
\end{abstract}

\section{INTRODUCTION}

Actuator performance is one of the main technological shortcomings preventing the development of successful general-purpose robots. While mainstream electric, pneumatic, and hydraulic actuators are capable of power densities, force densities, and efficiencies far in excess of those of natural actuators (i.e. muscles), they rarely can achieve all of these properties simultaneously or during transients [1], [2]. Furthermore, traditional actuators work best at size and force scales well in excess of those associated with the human body, rendering most industrial robots unsafe and too large for human interaction [3]. As a result, bioinspired robots lag well behind in performance compared to the organisms inspiring them, and haptic interfaces tend to be weak compared with their human operators.

Biological muscle has several properties that make it particularly difficult to mimic with conventional actuators [4]. Muscle has a variable stiffness, allowing it to passively stabilize a joint, and is generally associated with compliant tendons capable of efficient mechanical energy storage. It has very low inertia, yet it can generate a very high force, sustained across a displacement of up to $40 \%$ of the muscle's length. Muscle does this while remaining relatively efficient and powerful, with efficiency during cyclic motion estimated between $10 \%$ and $40 \%$ [5] and power densities near $100 \mathrm{~W} / \mathrm{kg}$.

A common approach to the problem of making robotic actuators behave more like biological muscle has been the development of artificial muscle materials, capable of undergoing shape change in response to electrical, thermal, or chemical stimulus [6]. While some of these materials have exciting longterm prospects, at present they are only suitable for niche applications, and fundamental progress in materials science

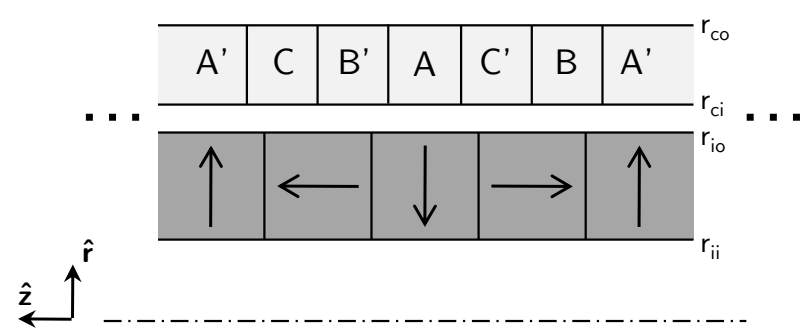

Fig. 1. The tubular motor configuration considered in this work is shown here, denoted the "HG" configuration. Nearest the center (indicated by a dotdashed line) is a quasi-Halbach magnet array, in which blocks with arrows are magnets, with the arrows indicating the direction of magnetization. The axial magnets in the array serve to provide closed flux paths, enhancing the field produced. Outside the magnets lies a coil, here shown arranged in a typical three-phase winding (A, B, and $\mathrm{C}$ denote phases, with negative current in the primed windings). This magnet and coil structure is repeated periodically along the $\mathrm{z}$-axis to form the motor. The radial dimensions in the model $\left(r_{i i}\right.$, $r_{i o}, r_{c i}$, and $\left.r_{c o}\right)$ are as labeled.

will be required to improve their performance to compete with natural muscle or even with conventional actuators.

Since artificial muscles are not yet able to improve upon conventional actuators, how can conventional actuators be improved to better compete with muscle? For the purposes of this work, we will focus on the potential development of electromagnetic (EM) actuators. In particular, the simplicity of direct-drive EM actuators is appealing as a canvas for improvements, and we will specifically discuss linear permanentmagnet (PM) direct drive actuators. Permanent magnet motors have favorable scaling properties [7], as do direct-drive linear actuators. While direct-drive motors are generally known for having low force densities and low efficiencies [2], their performance envelope is determined by their electromagnetic and thermal design, which can be modeled from basic physics, rather than by the tribological properties of gears, which are both difficult to model and difficult to improve.

At present, general models for the maximum theoretical performance of linear PM motors in terms of fundamental physics are unavailable [7], [8]; our objective will therefore be to develop such a general model, and to use it to find ways to improve the force capability and efficiency of these 
motors to a level competitive with biological muscle. Using our model, we will establish that direct-drive linear PM motors can be constructed with power densities, force densities, and efficiencies all simultaneously exceeding those of biological muscle. These improved properties can be achieved through a combination of active cooling, parts miniaturization, and careful electromagnetic design. We will show that the primary physical mechanism allowing this improved performance is the enhanced current density allowed by miniaturization and cooling, well in excess of that allowed by conventional motor design.

We will apply this model to determine the feasibility of direct-drive linear PM motors in a specific new application, as the wing actuators in bird-scale flapping-wing flying robots. Specifically, we will show the potential of such motors to sinusoidally drive a load of $1000 \mathrm{~N} / \mathrm{kg}$ at a peak velocity of $1 \mathrm{~m} / \mathrm{s}$ while retaining efficiencies above $40 \%$, as compared to the efficiency of just $10 \%$ to $20 \%$ for bird muscles under similar loading conditions [9], [10] .

\section{Fundamental Motor Physics}

In essence, we can consider a PM motor as an active material actuator in which a body force is produced on a conductor (the "active material") proportional to the surrounding magnetic flux density, $B$, and to the applied current density, $J$. For this basic model, we will assume that the magnetic field is produced "for free", consider it as a material property, and neglect the magnets necessary to produce it in a real motor. We will likewise ignore any practical restrictions on the provision of current, and assume that the conductor can be excited by a spatially uniform current density. Thus, the 0-D motor model consists of a bare conductor carrying a uniform current density immersed in a uniform magnetic field that is exactly perpendicular to the current. While this model can describe rotary and linear motors equally well, we will restrict our analysis to that of linear motors.

The force production and power consumption of a PM actuator, its basic input-output relations, are given by the Lorentz force law and by Ohm's law,

$$
\begin{aligned}
& F=B J V \text { and } \\
& P=\frac{J^{2} V}{\sigma},
\end{aligned}
$$

where $F$ is the force produced by the motor, $V$ is the volume of active material, $P$ is the electrical power converted by the motor into heat, and $\sigma$ is the conductivity of the active material. Here, we do not consider superconducting motors, which have zero Ohmic losses but instead have other power loss mechanisms and require energy-hungry cryogenics. Both of these quantities are directly proportional to the amount of active material-depending on the application, it may be preferable to describe this amount in terms of volume or in terms of mass. For this analysis, we will consider masssensitive applications; an alternative formulation in terms of volume can be straightforwardly derived.
We would like to describe motor performance in terms of parameters that do not couple motor size and drive current. One such parameter is the ratio of the force generated to the square root of the power consumed $\epsilon$, commonly known as the motor constant and listed on motor datasheets as $K_{m}$. The force generated per unit mass $F^{\prime}$, which we will call the force density, is another such parameter:

$$
\begin{gathered}
F^{\prime}=\frac{B J}{\rho_{c}}, \\
\epsilon \equiv \frac{F}{\sqrt{P}}=B \sqrt{\frac{\sigma M}{\rho_{c}} .}
\end{gathered}
$$

Here, $\rho_{c}$ is the mass density of the active material and $M$ is the total mass of active material. Note that each performance parameter is independently related to a single motor design parameter, in addition to the material properties $(B, \sigma$, and $\left.\rho_{c}\right)$ : the force density achievable depends on the maximum allowable current density, while the motor constant depends on the size of the motor. The maximum current density, in turn, is restricted either by the maximum amount of heat that can be removed from and tolerated by the motor or by non-thermal damage mechanisms (demagnetization and electromigration).

We can, in turn, relate these performance parameters to the energy efficiency of a linear PM motor operating on a given work loop. Consider perhaps the simplest situation, in which an actuator of total stroke $\ell_{s}$ sinusoidally drives an ideal dashpot at a frequency $\omega$, with a resulting peak force amplitude of $F_{0}$. In this case, the instantaneous mechanical power $P_{m}$ and electrical power $P_{e}$ are given by

$$
\begin{aligned}
P_{m} & =\frac{\omega \ell_{s} F_{0}}{2} \cos ^{2} \omega t, \\
P_{e} & =P_{m}+\frac{F_{0}^{2}}{\epsilon^{2}} \cos ^{2} \omega t .
\end{aligned}
$$

We can then integrate over one period in time to find the mechanical and electrical work per cycle $W_{m}$ and $W_{e}$, respectively, calculate the efficiency $\eta=W_{m} / W_{e}$, and define a dimensionless efficiency parameter $\hat{\eta}$

$$
\begin{gathered}
\eta=\frac{1}{1+\hat{\eta}}, \\
\hat{\eta} \equiv \frac{2 F_{0}}{\omega \ell_{s} \epsilon^{2}}=\underbrace{\frac{\rho_{c}}{\sigma B^{2}}}_{\text {materials }} \times \underbrace{\frac{2}{\omega \ell_{s}}}_{\text {velocity }} \times \underbrace{F_{0}^{\prime}}_{\begin{array}{c}
\text { force } \\
\text { density }
\end{array}}
\end{gathered}
$$

Here, we have broken out the components of $\epsilon$ to explain the scaling of $\hat{\eta}$, and $F_{0}^{\prime}$ denotes the peak force amplitude $F_{0}$ divided by the actuator mass $M$. Three factors are seen to contribute to the efficiency parameter: the first is related to the material properties of the actuator, the second is equal to the inverse of the peak actuator velocity, and the third is equal to the peak force density required by the work loop.

At first glance, the expression for $\hat{\eta}$ in Equation $6 \mathrm{~b}$ is disappointing, as it links an increase in force density directly to a decrease in efficiency. However, the efficiency remains high for even relatively large values of force density if copper 
and the best available magnets (1.47 $\mathrm{T}$ remanence $\mathrm{NdFeB})$ are used. For reference, it is estimated [10] that bird flight is less than $20 \%$ efficient. Using work loop parameters similar to those seen by pigeon flight muscles [9] $(2 / \omega L=1 \mathrm{~m} / \mathrm{s}$, $F_{0}^{\prime}=1000 \mathrm{~N} / \mathrm{kg}$ ) we find $\hat{\eta}=7.1 \times 10^{-2}$, or that the efficiency is $93 \%$. Thus, the actuator properties of copper wire in a magnetic field are sufficient to achieve very high efficiency even when operating under the relatively slow, heavily-loaded conditions of flapping-wing flight. While we anticipate that the capability of a real motor will be greatly reduced due to the need for magnets and spatially-varying fields, there is a considerable performance margin available.

\section{Actuator AnAlytical Modeling AND OPTIMIZATION}

\section{A. Tubular Motor Electromagnetic Model}

In order to model a real motor, we need to account for the magnetic field that can be produced by a given set of magnets, rather than assume a field provided by the environment. Fortunately for this process, modern rare-earth magnet materials behave linearly within their performance envelope [11], and so their fields can be computed from Poisson's equation. For maximum flexibility and ease of computation, we will consider linear motors with periodic magnet and coil structures, referred to as permanent magnet synchronous motors in the literature; the behavior of aperiodic motors such as voice coils can be derived from that of periodic motors by accounting for end effects [12], [13].

The magnets and coils in a linear motor can be arranged in many different ways [14], each amenable to a different set of analytical techniques. Here, we will consider a tubular motor configuration due to its efficient copper usage and intrinsic symmetry. Computation of the magnetic fields and resulting performance of tubular motors has been performed in many different ways [15]-[17], but the solutions reported in the literature tend to have numerical stability problems, and are presented in a manner that can make direct comparison between different configurations and different scales difficult.

In this work, we chose to directly solve Poisson's equation in cylindrical coordinates for several different motor topologies. Here we will present the analysis for a motor comprising a quasi-Halbach magnet array arranged to project a magnetic field radially outwards, surrounded by a layer of coils, as shown in Figure 1 and referred to henceforth as the "HG" configuration (Halbach-Gap). A Halbach array [11], [18] is chosen to produce closed magnetic flux paths, and thus strong fields, without using iron. An ideal Halbach array has a continuously varying direction of magnetization, which is very difficult to fabricate, but a segmented array (quasi-Halbach) reaches $90 \%$ of the ideal performance with only four uniform magnets per period [18]. While the $\mathrm{HG}$ configuration is relatively ineffective, we consider it here due to the relative brevity of its field model, and easy extension to a more-efficient structure with Halbach arrays on both sides of the air gap. Results for other magnet configurations, including configurations with iron components and completely closed flux paths, will be presented in a future publication.

The magnetic field can be determined by first breaking down the magnetization into a Fourier series:

$$
\begin{gathered}
\boldsymbol{M}=\sum_{n \text { odd }}\left[M_{r n} \cos (n k z) \hat{\boldsymbol{r}}+M_{z n} \sin (n k z) \hat{\boldsymbol{z}}\right], \\
M_{r n}=\frac{4 B_{r}}{n \pi \mu_{0}} \sin \left(\frac{n \pi \delta}{2}\right), \\
M_{z n}=-\frac{4 B_{r}}{n \pi \mu_{0}} \cos \left(\frac{n \pi \delta}{2}\right) .
\end{gathered}
$$

In these equations, $M$ is the magnetization vector, $M_{r n}$ and $M_{z n}$ are the radial and axial components of the magnetization, respectively, $n$ is the harmonic order, $k$ is the wavenumber of the magnet array (equal to $2 \pi$ divided by the pole pitch $p$ ), $B_{r}$ is the remanence of the permanent magnet material, and $\delta$ is the fractional width of the radial magnets in the array. Note that the magnetization, and thus the overall magnetic field, has only odd harmonics. The current density in the coil region can similarly be described in terms of a (scalar) Fourier series.

With the magnetization and current density described thusly, we can proceed to solve the appropriate form of Poisson's equation in the magnet, coil, and air regions of the motor:

$$
\begin{aligned}
\nabla^{2} \boldsymbol{A}_{\text {magnet }} & =-\mu_{0} \nabla \times \boldsymbol{M}, \\
\nabla^{2} \boldsymbol{A}_{\text {coil }} & =-\mu_{0} J_{n} \cos (n k z) \hat{\boldsymbol{\theta}}, \\
\nabla^{2} \boldsymbol{A}_{\text {air }} & =\mathbf{0},
\end{aligned}
$$

where $\boldsymbol{A}$ is the magnetic vector potential and $J_{n}$ is the Fourier series coefficient of the current density in the circumferential direction. Here, we have assumed that both the magnet and the conductor have unity relative permeability. The solution in the air regions is simply a linear combination of modified Bessel functions, but solution of the equations in the magnet and coil regions leads to inhomogeneous Bessel equations,

$$
\frac{\partial^{2} A}{\partial r}+\frac{1}{r} \frac{\partial A}{\partial r}-\left(c^{2}+\frac{1}{r^{2}}\right) A(r)=d,
$$

where $A$ is a function of the radial coordinate only and $c$ and $d$ are constants. These equations are often solved in the literature using Green's functions [15] or by approximating the radial magnets' magnetization as being nonuniform in order to give a solution in terms of elementary functions [16]. However, there exists a set of named functions that solve this equation, the modified Struve functions [17], [19]. So long as care is taken in the formulation of the solutions to avoid numerical stability problems, the modified Struve functions can be efficiently calculated using the power series and asymptotic series presented in [19], [20].

Using the modified Struve functions $\mathrm{L}_{0}$ and $\mathrm{L}_{1}$, the magnetic field in each region can be found, as shown in Equations 10. Here, $\mathrm{I}_{0}$ and $\mathrm{I}_{1}$ refer to modified Bessel functions of the first kind, $K_{0}$ and $K_{1}$ refer to modified Bessel functions of the second kind, $a$ and $b$ are arbitrary constants, and $\boldsymbol{H}$ is the magnetic field vector. These solutions must then be patched together using appropriate boundary conditions for the 
magnetic field and the magnetic flux density to find values for the constants $a$ and $b$ in each region, and to thus find the magnetic field throughout the motor structure.

To calculate the force produced by the motor, we can first compute the Maxwell stress tensor at the inner and outer surfaces of the coil region. We can then multiply the stress at the inner and outer coil surfaces by the appropriate circumferences, add them, and average over the length of a pole pitch to find the force per unit length generated by the motor. We can approximate the force production as being done by only the first spatial harmonics of the current and magnetic field distributions [18], greatly simplifying the calculation process. By gathering all of the dimensional parameters together, we find an expression for the total force generated by the motor,

$$
\begin{gathered}
F=\frac{B_{r} J_{1} \ell}{k^{2}} \hat{f}, \\
\hat{f} \equiv \pi\left(\hat{a}_{c} \hat{b}_{m}+\hat{a}_{m} \hat{b}_{c}\right),
\end{gathered}
$$

where $\hat{f}$ is given by Equation 11b. Here, $\ell$ is the overall length of the motor, $J_{1}$ is the first harmonic of the current density, and $\hat{f}$ is a dimensionless parameter that depends only on ratios between the pole pitch and the radii of the motor components.

The dimensionless force $\hat{f}$ depends on four dimensionless parameters related to the magnetic fields: $\hat{a}_{c}$ and $\hat{b}_{c}$, describing the coil, and $\hat{a}_{m}$ and $\hat{b}_{m}$, describing the magnets. $\hat{a}_{c}$ and $\hat{b}_{c}$, given below in Equations 12a and 12b, do not depend upon the particular arrangement of magnets, but the dimensionless magnet parameters $\hat{a}_{m}$ and $\hat{b}_{m}$ must be found and tabulated for each magnet configuration by solving the magnetic field boundary conditions. In Equations $12 \mathrm{a}$ and $12 \mathrm{~b}, r_{c i}$ is the coil inner radius, and $r_{c o}$ is the coil outer radius.

$$
\begin{aligned}
\hat{a}_{c}=k r_{c i}[ & \left(\mathrm{I}_{1}\left(k r_{c i}\right)-\mathrm{L}_{1}\left(k r_{c i}\right)\right) \mathrm{K}_{0}\left(k r_{c i}\right)+ \\
& \left.\left(\mathrm{I}_{0}\left(k r_{c i}\right)-\mathrm{L}_{0}\left(k r_{c i}\right)\right) \mathrm{K}_{1}\left(k r_{c i}\right)\right]- \\
k r_{c o}[ & \left(\mathrm{I}_{1}\left(k r_{c o}\right)-\mathrm{L}_{1}\left(k r_{c o}\right)\right) \mathrm{K}_{0}\left(k r_{c o}\right)+ \\
& \left.\left(\mathrm{I}_{0}\left(k r_{c o}\right)-\mathrm{L}_{0}\left(k r_{c o}\right)\right) \mathrm{K}_{1}\left(k r_{c o}\right)\right], \\
\hat{b}_{c}=k r_{c i}[ & \left(\mathrm{I}_{0}\left(k r_{c i}\right)-\mathrm{L}_{0}\left(k r_{c i}\right)\right) \mathrm{I}_{1}\left(k r_{c i}\right)- \\
& \left.\left(\mathrm{I}_{1}\left(k r_{c i}\right)-\mathrm{L}_{1}\left(k r_{c i}\right)\right) \mathrm{I}_{0}\left(k r_{c i}\right)\right]- \\
k r_{c o}[ & \left(\mathrm{I}_{0}\left(k r_{c o}\right)-\mathrm{L}_{0}\left(k r_{c o}\right)\right) \mathrm{I}_{1}\left(k r_{c o}\right)- \\
& \left.\left(\mathrm{I}_{1}\left(k r_{c o}\right)-\mathrm{L}_{1}\left(k r_{c o}\right)\right) \mathrm{I}_{0}\left(k r_{c o}\right)\right] .
\end{aligned}
$$

For the HG configuration, $\hat{a}_{m}$ and $\hat{b}_{m}$ are given by Equations $13 \mathrm{a}$ and $13 \mathrm{~b}$. The dimensionless magnet parameters, in this case, depend upon $r_{i i}$, the inner radius of the magnets inside the coil, and $r_{i o}$, the outer radius of the magnets inside the coil. Note that all radii are non-dimensionalized by the wavenumber $k$.

The remaining basic performance measures for the motor can also now be written:

$$
\begin{aligned}
P & =\frac{\ell J_{1}^{2}}{\sigma k^{2}} \hat{P}, \\
M & =\frac{\rho_{c} \ell}{k^{2}} \hat{m} .
\end{aligned}
$$

$$
\begin{aligned}
& \boldsymbol{H}_{\text {coil }}=\sum_{n \text { odd }} {\left[a \mathrm{I}_{1}(n k r)+b \mathrm{~K}_{1}(n k r)-\frac{\pi J_{n}}{2 n k}\left(\mathrm{I}_{1}(n k r)-\mathrm{L}_{1}(n k r)\right)\right] \hat{\boldsymbol{r}} \sin (n k z)+} \\
& {\left[-a \mathrm{I}_{0}(n k r)+b \mathrm{~K}_{0}(n k r)+\frac{\pi J_{n}}{2 n k}\left(\mathrm{I}_{0}(n k r)-\mathrm{L}_{0}(n k r)\right)\right] \hat{\boldsymbol{z}} \cos (n k z), } \\
& \boldsymbol{H}_{\text {air }}=\sum_{n \text { odd }}\left[a \mathrm{I}_{1}(n k r)+b \mathrm{~K}_{1}(n k r)\right] \hat{\boldsymbol{r}} \cos (n k z)+\left[-a \mathrm{I}_{0}(n k r)+b \mathrm{~K}_{0}(n k r)\right] \hat{\boldsymbol{z}} \sin (n k z) .
\end{aligned}
$$$$
\hat{a}_{m}=0
$$$$
\hat{b}_{m}=\frac{\pi}{2} \sin \left(\frac{\pi \delta}{2}\right)\left[k r_{i i}\left(\left(\mathrm{I}_{0}\left(k r_{i i}\right)-\mathrm{L}_{0}\left(k r_{i i}\right)\right) \mathrm{I}_{1}\left(k r_{i i}\right)-\left(\mathrm{I}_{1}\left(k r_{i i}\right)-\mathrm{L}_{1}\left(k r_{i i}\right)\right) \mathrm{I}_{0}\left(k r_{i i}\right)\right)-\right.
$$$$
\left.k r_{i o}\left(\left(\mathrm{I}_{0}\left(k r_{i o}\right)-\mathrm{L}_{0}\left(k r_{i o}\right)\right) \mathrm{I}_{1}\left(k r_{i o}\right)-\left(\mathrm{I}_{1}\left(k r_{i o}\right)-\mathrm{L}_{1}\left(k r_{i o}\right)\right) \mathrm{I}_{0}\left(k r_{i o}\right)\right)\right]+
$$$$
\cos \left(\frac{\pi \delta}{2}\right)\left[k r_{i o} \mathrm{I}_{1}\left(k r_{i o}\right)-k r_{i i} \mathrm{I}_{1}\left(k r_{i i}\right)\right]
$$ 
where $\hat{P}$ is the dimensionless power parameter and $\hat{m}$ is the dimensionless mass parameter. The dimensionless power parameter is independent of motor configuration, while the dimensionless mass parameter changes with both the motor configuration and the motor materials. For example, in Equation 17 below, the dimensionless mass parameter is given for the HG configuration:

$$
\begin{aligned}
& \hat{P} \equiv \frac{\pi}{2}\left(\left(k r_{c o}\right)^{2}-\left(k r_{c i}\right)^{2}\right) \\
& \hat{m}=\pi\left[\frac{\rho_{m}}{\rho_{c}}\left(\left(k r_{i o}\right)^{2}-\left(k r_{i i}\right)^{2}\right)+\left(k r_{c o}\right)^{2}-\left(k r_{c i}\right)^{2}\right]
\end{aligned}
$$

where $\rho_{m}$ is the mass density of the magnet. We can now also compute the motor constant $\epsilon$,

$$
\begin{gathered}
F^{\prime}=\frac{B_{r} J_{1}}{\rho_{c}} \frac{\hat{f}}{\hat{m}}, \\
\epsilon=B_{r} \hat{\epsilon} \sqrt{\frac{\sigma M}{\rho_{c}}}, \quad \hat{\epsilon} \equiv \frac{\hat{f}}{\sqrt{\hat{P} \hat{m}}} .
\end{gathered}
$$

Note that the expressions for the motor constant and the force density are almost identical to those presented in Section II, with the addition of dimensionless correction factors $\hat{\epsilon}$ and $\hat{f} / \hat{m}$ representing the effects of the spatially varying magnetic fields and the mass of the magnets. The functional form of Equations 19 and 18 do not depend on the details of the magnet and conductor configuration; the motor configuration and geometry affect only the value of the dimensionless correction factors.

The dimensionless motor constant $\hat{\epsilon}$ depends only on the ratios of the layer radii to the pole pitch, and can be shown to be convex in these dimensionless lengths. There is a broad optimum range of geometry for the HG magnet configuration, near dimensionless geometry $k r_{i i}=0.86, k r_{i o}=3.0, k r_{c i}=$ $3.03, k r_{c o}=3.87$, and $\delta=0.41$. (An air gap between the magnets and coils equal to $1 \%$ of the radius is included as a thermal expansion and manufacturing tolerance allowance.) With this geometry, the maximum value of the dimensionless motor constant is $\hat{\epsilon}=0.21$. Other configurations yield better optimum values for the dimensionless motor constant; for instance the results of Trumper et al. for a planar Halbach array motor configuration [18] can be transformed into this framework to give a value of $\hat{\epsilon}=0.37$.

\section{B. Finite Element Validation of Electromagnetic Model}

In order to check the accuracy of our analytical solution for the motor magnetic fields, we performed a finite element (FEA) simulation of the optimum HG motor configuration given in the previous section and compared the results to those derived analytically for an identical motor. The FEA was performed using a remanence of $1.47 \mathrm{~T}$ and a magnet relative permeability of 1.05 in ANSYS. Special attention to numerical instabilities that arise in the analytical model when the value of $n k r$ exceeds 30 at the outside of the motor is needed to analytically compute the magnetic field to high harmonic order. Contour plots of the radial magnetic flux density are shown in Figure 2, for both the analytical model of Equations 10 and the FEA model. These plots show good qualitative agreement, primarily differing due to the inherent limitations of a Fourier series representation for the discontinuous magnetization.

FEA was also used to make quantitative assessments of the analytical solution's veracity. Figure 3 shows a comparison of the radial flux density in the middle of the air gap between the analytical and FEA models, with approximately $8 \%$ discrepancy. This difference can be explained primarily by the non-unity relative permeability of the magnets in the FEA model. For a slightly different motor geometry, the linear force density under a square wave current density excitation was computed using both analytical and FEA techniques; the analytical method predicted a force between $1 \%$ and $3 \%$ lower than that from the FEA.

\section{Thermal Model}

While Equation 19 describes the efficiency of the motor while operating under any conditions and at any size scale, we need to specify the maximum current density $J_{1}$ in order to determine the achievable force density. The dominant physical process limiting the current density is Joule heating of the wire and magnets beyond their maximum operating temperatures. In order to specify the maximum current density, then, we can use a basic heat transfer model [21] to relate it to the maximum temperature rise $\Delta T$.

For brevity, consider a simple, planar heat transfer model for the HG motor configuration in which it is cooled solely by convection on its exterior, as characterized by a convection coefficient $\bar{h}$. From elementary heat transfer theory, we can calculate a thermal resistance, $R_{t h}$ from the inner surface of the coil to the ambient according to

$$
R_{t h}=\frac{r_{c o}-r_{c i}+2 \kappa_{c} \bar{h}^{-1}}{4 \pi \kappa_{c} r_{c o}},
$$

where $\kappa_{c}$ is the thermal conductivity of the coil. We can then proceed to calculate the motor temperature as a function of the time-average (RMS) current density $\bar{J}_{1}$,

$$
\begin{gathered}
\Delta T=\frac{\pi\left(r_{c, o}^{2}-r_{c, i}^{2}\right) R_{t h} \bar{J}_{1}^{2}}{2 \sigma}=\frac{\bar{J}_{1}^{2}}{\sigma \kappa_{c} k^{2}} \hat{P} \hat{R}_{t h} \\
\hat{R}_{t h} \equiv \pi \kappa_{c} R_{t h}=\frac{k r_{c o}-k r_{c i}+4 \pi \mathrm{Bi}^{-1}}{4 k r_{c o}}, \\
\mathrm{Bi} \equiv \frac{p \bar{h}}{\kappa_{c}}
\end{gathered}
$$

where we have defined a Biot number $\mathrm{Bi}$ in terms of the pole pitch $p$. Combining this expression (Equation 21a) with Equation 18 and the definition of $\hat{\epsilon}$, we find the perhaps surprising result that the thermal constraint is equivalent to a constraint on the pole pitch:

$$
p \leq \frac{2 \pi B_{r}}{\rho_{c} \bar{F}^{\prime}} \sqrt{\pi \sigma \kappa_{c} \Delta T} \times \frac{\hat{\epsilon}}{\sqrt{\hat{m} \hat{R}_{t h}}},
$$

where $\bar{F}^{\prime}$ denotes the RMS force density. This relationship suggests that it should be possible to achieve an arbitrarily 

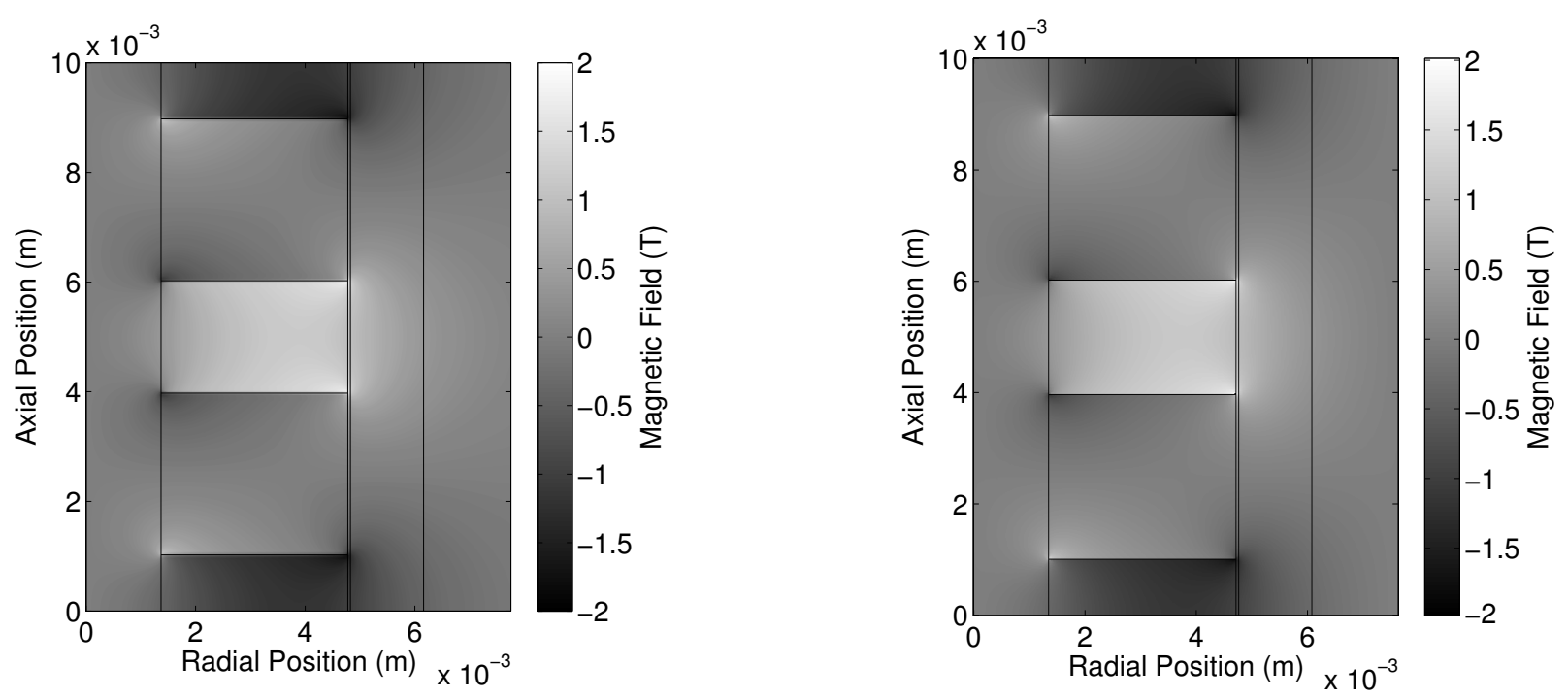

Fig. 2. A plot of the radial magnetic flux density for an optimized HG configuration motor $\left(k r_{i i}=0.86, k r_{i o}=3.0, k r_{c i}=3.03, k r_{c o}=3.87, \delta=0.41\right)$ as computed using ANSYS finite element software (right) and using the analytical model of Equations 10 with 75 harmonic terms (left). Qualitative agreement between the analytical and numerical flux plots is excellent, primarily differing in the sharpness of the boundaries between radial and axial magnets.

high force density so long as the pole pitch is made sufficiently small. While this is contrary to standard motor design practice, which is done by simply assuming a maximum current density (typically $10^{7} \mathrm{~A} / \mathrm{m}^{2}$ ), it is in agreement with the scaling described by Cugat et al. [7] and can be intuitively explained with reference to microelectronics, where current densities over $10^{9} \mathrm{~A} / \mathrm{m}^{2}$ are routinely used in small bond wires and interconnects.

As a numerical example consider once again the work loop parameters associated with pigeon flight muscles [9], which produce an RMS force density $\bar{F}^{\prime} \approx 700 \mathrm{~N} / \mathrm{kg}$, the optimum geometry for the HG configuration, a coil thermal conductivity of $\kappa_{c} \approx 1 \mathrm{~W} / \mathrm{m} \cdot \mathrm{K}$, and a maximum temperature rise of $100^{\circ} \mathrm{C}$. If we wish to have a pole pitch of greater than $10 \mathrm{~mm}$, we will require a heat transfer coefficient $\bar{h} \geq 400 \mathrm{~W} / \mathrm{m}^{2} \mathrm{~K}$. This heat transfer coefficient can be achieved through vigorous air cooling or by simple liquid cooling [21].

\section{Model Summary and Application}

Just as in the analysis of the ideal motor in Section II, certain basic scaling properties of tubular linear synchronous motors are readily apparent. The motor constant $\epsilon$ in Equation 19 scales according to the same parameters as it did in Equation 4, with an additional dimensionless parameter describing the deviations from the ideal motor. Thus, even with the permanent magnet structure included, the motor constant still scales with the square root of the motor mass.

We can also examine the impact of the dimensionless motor constant correction factor on the motor efficiency,

$$
\hat{\eta}=\underbrace{\frac{\rho_{c}}{\sigma B_{r}^{2}}}_{\text {materials }} \times \underbrace{\frac{2}{\omega \ell_{s}}}_{\text {velocity }} \times \underbrace{F_{0}^{\prime}}_{\begin{array}{c}
\text { force } \\
\text { density }
\end{array}} \times \underbrace{\hat{\epsilon}^{-2}}_{\begin{array}{c}
\text { motor } \\
\text { geometry }
\end{array}}
$$

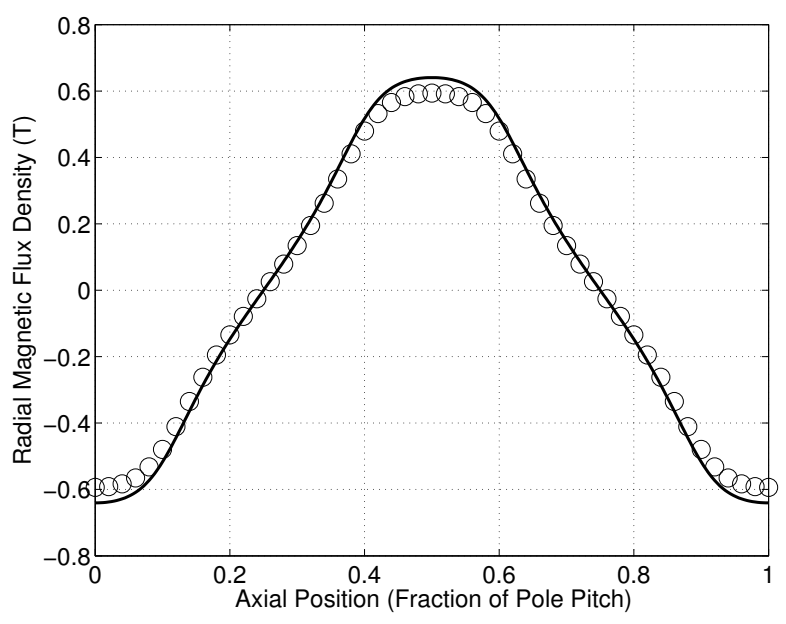

Fig. 3. A comparison of the radial magnetic flux density in the center of the coil of the HG motor depicted in Figure 2 between the 75-harmonic analytical model (solid line) and the FEA model (open circles) The maximum deviation occurs near the peak of the flux waveform, where the analytical solution is approximately $8 \%$ greater in magnitude than the FEA result.

Here, we can see that $\hat{\epsilon}$ is a dimensionless efficiency parameter that can be used to compare the efficiencies of different motor configurations and geometric ratios at any size scale. We have shown that, for the tubular HG configuration, the optimum $\hat{\epsilon}=0.21$, and we can use the results of other magnetic field calculations to find the optimum $\hat{\epsilon}$ for additional configurations. In some literature [22] a similar parameter to $\hat{\epsilon}$, the "motor constant square density" is used to characterize motor performance, but this parameter does not separate the influence of material selection from that of geometric design.

The overall size of the motor components scales with the 


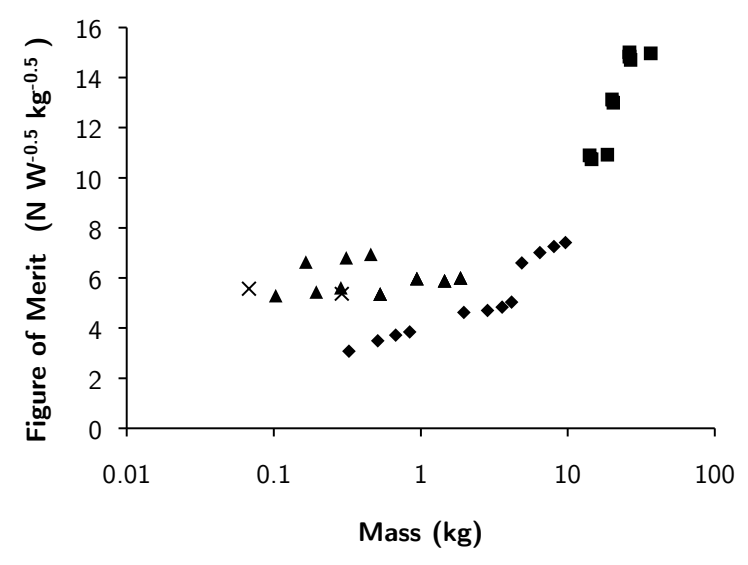

Fig. 4. The ratio of the motor constant to the square root of the mass is plotted for 30 different linear synchronous motors from four different manufacturers: Copley (diamonds), Moog (squares), Anorad (triangles), and Faulhaber (crosses). With the exception of the Moog motors, this figure of merit is independent of size. Compare with the theoretical figure of merit for an $\mathrm{HG}$ motor of $26 \mathrm{~N} \cdot \mathrm{W}^{-0.5} \cdot \mathrm{kg}^{-0.5}$.

pole pitch, and thus with the required force density:

$$
p \leq 2 \pi \underbrace{\frac{B_{r} \sqrt{\pi \sigma \kappa_{c}}}{\rho_{c}}}_{\text {materials }} \times \underbrace{\sqrt{\Delta T}}_{\begin{array}{c}
\text { thermal } \\
\text { limit }
\end{array}} \times \underbrace{\frac{1}{\bar{F}^{\prime}}}_{\begin{array}{c}
\text { force } \\
\text { density }
\end{array}} \times \underbrace{\sqrt{\frac{\hat{\epsilon}}{\hat{m} \hat{R}_{t h}}}}_{\begin{array}{c}
\text { motor } \\
\text { geometry }
\end{array}} .
$$

If internal thermal conduction limits the heat transfer out of the motor, the pole pitch scales inversely with the required force density. If convective thermal resistance dominates heat transfer out of the motor, the required pole pitch scales as the inverse square of the specified force density-high force densities call for highly miniaturized coils and magnetic structures, which may be difficult to fabricate. Short pole pitches also require long motors, with many repeating units and thus a high part and/or winding count.

Since the only motor dimensions that appear outside the dimensionless parameters are the pole pitch and overall length, the effect of the radial geometry of any motor configuration is universal and independent of the desired performance. Thus, the radial dimensions can be optimized for each configuration to yield the highest possible value of the dimensionless motor constant $\hat{\epsilon}$. The use of a closed-form analytical model renders geometric optimization very computationally efficient, and a modest computer proves capable of examining thousands of geometries per second. Furthermore, $\hat{\epsilon}$ is convex in most geometric parameters, although some configurations perform best as the overall radial scale tends towards infinity (i.e. a flat plate rather than a tube.)

\section{E. Comparison with Commercial Motors}

While motor manufacturers do not release enough data for a full evaluation of Equations 23 and 24, we can nonetheless verify the general scaling of the model against existing motors. The ratio $\epsilon / \sqrt{M}$, which should depend only on motor internal

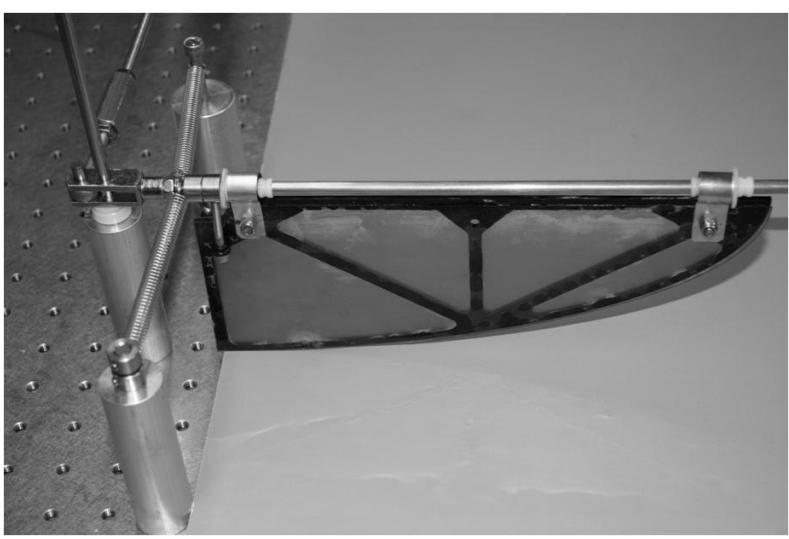

Fig. 5. A photograph of the prototype wing-flapping test-bed, showing the polyimide film wing, the brass wing spar, and the actuator attachment point. The wing has a span of $250 \mathrm{~mm}$, and is connected to the actuator with a 10 $\mathrm{mm}$ moment arm. The springs connected to the wing spar have a very low stiffness, and are used solely to keep the wing centered in its range of motion. Not shown are the actuator under test or the LVDT position transducer.

geometry and on materials choice, is plotted for a variety of commercially-available linear synchronous motors across three orders of magnitude in size in Figure 4. With the exception of the largest motors, which incorporate more mechanical components than the others, this ratio is approximately constant for each motor type. There is considerable variability in motor performance, however, with this figure of merit varying by a factor of three between different motor types. By comparison, with $1.47 \mathrm{~T}$ magnets and an optimum HG geometry, the model of this work suggests a figure of merit of $26 \mathrm{~N} \cdot \mathrm{W}^{-0.5} \cdot \mathrm{kg}^{-0.5}$, about twice as high as the large Moog motors can offer. The difference can be ascribed to the mechanical components of the Moog motor (bearings, sealed housing, etc.) and certain non-idealities of motor design not covered by this model (copper packing factor, end effects, etc.).

A similar examination of the pole pitch and force density of commercial motors verifies the scaling of Equation 24. For example, Anorad's passively-cooled $15 \mathrm{~mm}$ pole pitch motors average $80 \mathrm{~N} / \mathrm{kg}$ while their $30 \mathrm{~mm}$ motors average $55 \mathrm{~N} / \mathrm{kg}$, in close agreement with the expected scaling for convectiondominated thermal resistance. Active cooling in these motors results in very modest improvements in force density, perhaps due to poor coupling between the cooling fluid and the coils.

\section{ApPliCATIONS TO FlapPing Flight}

Our motor model gives us a way to determine the size, complexity, and cooling requirements of a linear PM motor for any given performance requirements, including requirements traditionally [1] thought outside the capabilities of such motors. As we have considered previously in this work, flapping-wing flight is a problem in robotics that is particularly constrained by actuator performance. The limited performance of conventional actuators is usually overcome by driving the wings symmetrically with a single high-speed rotary motor or engine and a gear reduction system, but this design does not 
allow the asymmetric flapping that is essential to low-speed maneuverability [23]. To achieve full control authority over the robot's trajectory, each wing needs to be actuated independently with variable stroke. The simplest way to achieve this is to use direct-drive linear actuators to move each wing.

Using Equation 23, we can determine the efficiency achievable with motors in such an actuation scheme. If we assume that motors of an HG configuration will be used, and continue to match the properties of the pigeon pectoralis muscle, we find that the efficiency of our hypothetical two-actuator directdrive bird robot will be $40 \%$, well in excess of the efficiency of birds in nature.

In order to further explore this application, we have begun construction of a flapping wing actuator test-bed, shown in Figure 5. A wing with a passive feathering axis is connected to a conventional linear EM actuator (BEI Kimco LA25-42000A) with similar dimensions and geometry to a pigeon wing. This apparatus will be used to test motors designed and built according to the principles described in this work, and to obtain suitable comparison data using off-the-shelf actuators.

\section{DISCUSSION AND FUTURE WORK}

In conclusion, by developing a non-dimensionalized model for linear PM motor performance, we have established that linear motors are capable of extremely high, to date unrealized performance. With suitable provision of active cooling and miniaturized components, linear PM motors can achieve force densities more than an order of magnitude higher than those of commercially-available motors while retaining acceptable efficiency. This capability opens up a large range of new applications in robotics, from locomotion and manipulation to haptic interfaces, at the relatively small cost of requiring custom motor design for each application.

To obtain a more accurate estimate of motor performance, our analysis framework can be modified to include nonidealities such as the effects of less than $100 \%$ copper fill in the coils, imperfect coil winding factors, and overhung and underhung coil configurations. It can also be adapted to handle saturable iron flux return paths, to accurately describe magnets that are simpler in fabrication than Halbach arrays. We will also use the lessons taught by our model to construct a prototype motor to illustrate the theoretically achievable performance.

\section{REFERENCES}

[1] J. D. Madden, "Mobile robots: Motor challenges and materials solutions," Science, vol. 318, no. 5853, pp. 1094-1097, 2007.

[2] J. M. Hollerbach, I. W. Hunter, and J. Ballantyne, "A comparative analysis of actuator technologies for robotics," in The Robotics Review 2 (O. Khatib, J. J. Craig, and T. Lozano-Perez, eds.), (Cambridge, MA), pp. 299-342, MIT Press, 1992.

[3] A. Bicchi and G. Tonietti, "Fast and 'soft-arm' tactics," IEEE Robotics \& Automation Magazine, vol. 11, pp. 22-33, June 2004.

[4] F. E. Zajac, "Muscle and tendon: Properties, models, scaling, and application to biomechanics and motor control," Critical Reviews in Biomedical Engineering, vol. 17, no. 4, pp. 359-411, 1989.

[5] N. P. Smith, C. J. Barclay, and D. S. Loiselle, "The efficiency of muscle contraction," Progress in Biophysics and Molecular Biology, vol. 88, no. 1 , pp. $1-58,2005$.
[6] J. D. W. Madden, N. A. Vandesteeg, P. A. Anquetil, P. G. A. Madden, A. Takshi, R. Z. Pytel, S. R. Lafontaine, P. A. Wieringa, and I. W. Hunter, "Artificial muscle technology: physical principles and naval prospects," IEEE Journal of Oceanic Engineering, vol. 29, pp. 706-728, Jul. 2004.

[7] O. Cugat, J. Delamare, and G. Reyne, "Magnetic micro-actuators and systems (MAGMAS)," IEEE Transactions on Magnetics, vol. 39, pp. 3607-3612, Nov. 2003.

[8] E. R. Laithwaite, "The goodness of a machine," Proceedings of the IEE, vol. 112, pp. 538-542, Mar. 1965.

[9] A. A. Biewener, W. R. Corning, and B. W. Tobalske, "In vivo pectoralis muscle force-length behavior during level flight in pigeons (Columba livia)," Journal of Experimental Biology, vol. 201, pp. 3293-3307, 1998.

[10] A. Kvist, Å. Lindström, M. Green, T. Piersma, and G. H. Visser, "Carrying large fuel loads during sustained bird flight is cheaper than expected," Nature, vol. 413, pp. 730-732, Oct. 2001.

[11] K. Halbach, "Design of permanent multipole magnets with oriented rare earth cobalt material," Nuclear Instruments and Methods, vol. 169, pp. 1-10, 1980.

[12] M. G. Lee, S. Q. Lee, and D.-G. Gweon, "Analysis of Halbach magnet array and its application to linear motor," Mechatronics, vol. 14, pp. 115128, 2004.

[13] D. Golda and M. L. Culpepper, "Modeling 3D magnetic fields for precision magnetic actuators that use non-periodic magnet arrays," Precision Engineering, vol. 32, pp. 134-142, Apr. 2008.

[14] E. R. Laithwaite, "Linear electric machines - a personal view," Proceedings of the IEEE, vol. 63, pp. 250-290, Feb. 1975.

[15] J. Wang, G. W. Jewell, and D. Howe, "A general framework for the analysis and design of tubular linear permanent magnet machines," IEEE Transactions on Magnetics, vol. 35, pp. 1986-2000, May 1999.

[16] N. Bianchi, "Analytical field computation of a tubular permanent-magnet linear motor," IEEE Transactions on Magnetics, vol. 36, pp. 3798-3801, Sep. 2000.

[17] Wijono and H. Arof, "Open circuit field distribution and induced voltage of a cylindrical permanent magnet linear generator," American Journal of Applied Sciences, vol. 4, no. 11, pp. 912-917, 2007.

[18] D. L. Trumper, M. E. Williams, and T. H. Nguyen, "Magnet arrays for synchronous machines," in Conference Record of the 1993 IEEE Industry Applications Society Annual Meeting, pp. 9-18, Oct. 1993.

[19] M. Abramowitz and I. A. Stegun, eds., Handbook of Mathematical Functions with Formulas, Graphs, and Mathematical Tables. New York: Dover, tenth ed., 1964.

[20] S. Zhang and J. Jin, Computation of Special Functions. New York: Wiley, 1996.

[21] J. H. Lienhard, IV and J. H. Lienhard, V, A Heat Transfer Textbook. Cambridge, MA: Phlogiston Press, 3rd ed., 2008.

[22] T. Mizuno, M. Kawai, F. Tsuchiya, M. Kosugi, and H. Yamada, "An examination for increasing the motor constant of a cylindrical moving magnet-type linear actuator,' IEEE Transactions on Magnetics, vol. 41 pp. 3976-3978, Oct. 2005.

[23] B. W. Tobalske, "Biomechanics of bird flight," Journal of Experimental Biology, vol. 210, pp. 3135-3146, 2007. 